\title{
Primož Trubar, le « Luther slovène »
}

\author{
Jonatan Vinkler
}

\section{OpenEdition}

Journals

Édition électronique

URL : http://journals.openedition.org/rbnu/719

DOI : 10.4000/rbnu.719

ISSN : 2679-6104

\section{Éditeur}

Bibliothèque nationale et universitaire de Strasbourg

\section{Édition imprimée}

Date de publication : 1 novembre 2017

Pagination : 108-111

ISBN : 9782859230678

ISSN : 2109-2761

\section{Référence électronique}

Jonatan Vinkler, «Primož Trubar, le « Luther slovène » », La Revue de la BNU [En ligne], 16 | 2017, mis en ligne le 01 juillet 2019, consulté le 10 décembre 2020. URL : http://journals.openedition.org/rbnu/719 ; DOl : https://doi.org/10.4000/rbnu.719

\section{cc) (i) (2)}

La Revue de la BNU est mise à disposition selon les termes de la Licence Creative Commons Attribution - Pas d'Utilisation Commerciale - Partage dans les Mêmes Conditions 4.0 International. 


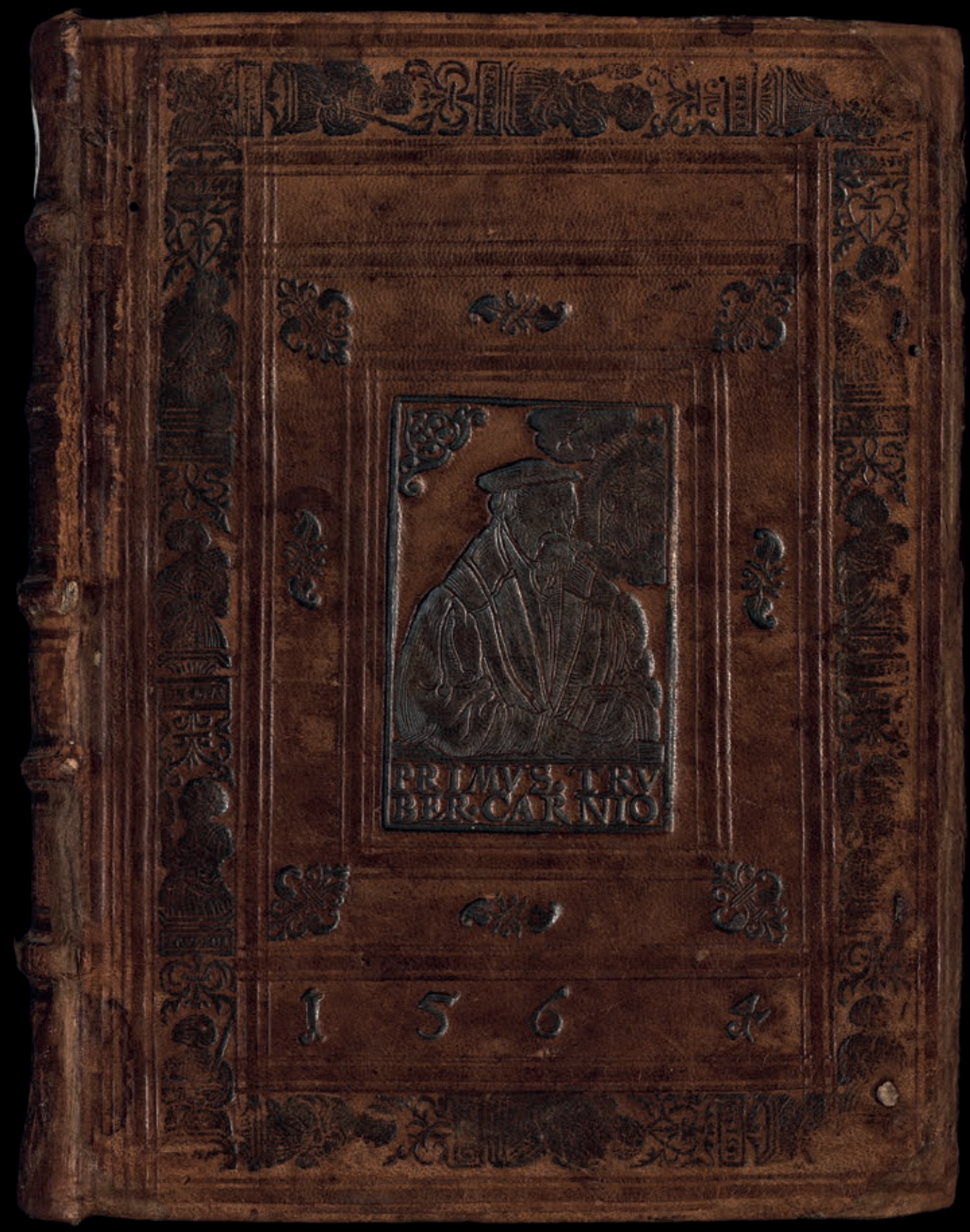

Portrait de Primož Trubar (Samuel Streler, 1561) sur le plat de reliure de l'ouvrage Postila, to jest, kratko istlmačenie vsih nedelskih' Evangeliov'... = Kurtze Auszlegung über die Sontags / und der fürnembsten Fest Evangelia..., Tübingen [i. e. Urach], [Ulrich Morhart], 1563. Il s'agit là du plus ancien portrait connu de Trubar. 


\section{$\overline{\text { SLOVÉNIE }}$ \\ PRIMOŽ TRUBAR, \\ LE « LUTHER SLOVÈNE »}

PAR JONATAN VINKLER

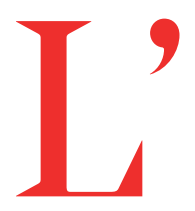

identité nationale slovène se définit, depuis la fin du $18^{\mathrm{e}}$ siècle, par identification avec la langue littéraire commune du pays et les œuvres littéraires rédigées en slovène. L'essor continu de la littérature et des belles-lettres trouve son origine dans l'introduction de la réforme luthérienne allemande chez les Slovènes au $16^{\mathrm{e}}$ siècle. La Réforme, «transposée " dans la scène linguistique slovène, est à l'origine du premier ouvrage connu dans cette langue - le Catechismus, publié en 1550 par Primož Trubar (1508-1586), un prêtre protestant. Avant cet ouvrage, le slovène n'avait été utilisé qu'à de rares occasions au Moyen Âge, à des fins liturgiques et administratives, comme le montrent des manuscrits médiévaux du $16^{\mathrm{e}}$ siècle (avec des témoignages à partir de 972 : Brižinski spomeniki, Rateški rokopis, Stiški rokopis, des certificats, etc.). Depuis la publication du Catechismus, la langue et la littérature slovènes n'ont cessé d'être utilisées et développées. À l'instar de Luther pour la langue et la littérature allemandes, Primož Trubar est considéré comme le pionnier de la langue et de la littérature slovènes. Issu d'un milieu rural et artisanal, il est né d'un père meunier dans le village de Raščica, théâtre d'importantes communications entre Ljubljana (dans le duché de Carniole) et Rijeka (Fiume). Trubar fut amené à voyager pendant sa scolarité et sa formation professionnelle. Il fréquenta dans un premier temps le lycée de glagolitique ${ }^{1}$ de Rijeka, puis l'école monastique Saint-Pierre de Salzbourg. Avant d'étudier à l'école de Saint-Étienne, à Vienne, il se rendit à Trieste, où il s'instruisit auprès de l'évêque italien Pietro Bonomo, célèbre humaniste, qui l'initia à la littérature humaniste contemporaine (Érasme de Rotterdam), ainsi qu'aux derniers écrits de la Réforme de Genève, par exemple l'Institutio christianae religionis de Jean Calvin. Malgré sa formation discontinue, Trubar réussit à acquérir des connaissances dépassant largement le niveau d'éducation d'une grande partie du clergé de son époque. Par ailleurs autodidacte zélé, il acquit les connaissances qu'il ne put apprendre à l'école grâce à l'étude acharnée d'un grand nombre d'ouvrages. Outre ses talents de musicien (il finança notamment son séjour à Salzbourg grâce au chant) et d'orateur, il utilisa aussi, dans le cadre de sa profession ecclésiastique - embrassée grâce à l'évêque Bonomo -, 
diverses langues : le croate (malgré des difficultés à lire le glagolitique), l'allemand, l'italien, le latin et le grec, ainsi que quelques mots de tchèque. Il fut un théologien catholique et protestant de renom, comme l'illustrent les nombreuses citations patristiques et de théologie protestante allemande que l'on retrouve dans ses ouvrages. Prêtre indépendant à partir de 1530, il commença à prêcher dans la paroisse de Sainte-Hélène, à Loka (Basse-Styrie). De là, il se rendit à Ljubljana, en passant par Laško et Celje. À partir de 1542, il obtint un canonicat à la cathédrale Saint-Nicolas, sous la direction de Franc Kacijanar, évêque de Ljubljana. Il conserva ce poste jusqu'en 1547, date à laquelle il dut fuir la ville pour échapper à la condamnation du nouvel évêque de Ljubljana, Urban Tekstor, qui l'accusait, ainsi que ses collaborateurs (P. Wiener, L. Mertlic, J. Dragolič et d'autres) d'enseigner une doctrine hérétique. Tekstor, qui fréquentait personnellement les plus hauts représentants de l'ordre des jésuites, fut le premier à appliquer les mesures du concile de Trente dans le diocèse de Ljubljana.

En 1548, Trubar se réfugie à Nuremberg, chez l'ami et collaborateur de Luther, Veit Dietrich; sous l'influence de ce dernier, il embrasse le protestantisme et obtient, grâce à ses recommandations, son premier emploi en tant que prédicateur protestant à Rothenburg ob der Tauber. C'est à cette époque qu'il commence à écrire et publie le premier livre en slovène. Trubar passe également du temps dans les villes allemandes de Kempten, Urach, Lauffen et Derendingen (aujourd'hui un quartier de Tübingen).

Les travaux de Trubar atteignent leur apogée dans les années 1560, lorsque la noblesse locale du duché de Carniole le nomme surintendant de cette province. Il devient alors le tout premier évêque protestant, représentant de l'Église évangélique, des pays slovénophones. Entre 1562 et 1565, Trubar fonde un réseau d'églises en Carniole et une école à Ljubljana, et se charge également de l'éducation des jeunes membres du clergé protestant (Dalmatin, Savinec, Steiner et d'autres, qui bénéficient de l'appui de l'université de Tübingen). Outre le Catechismus, il publie ses ouvrages symboliques les plus importants, intitulés Articuli (1562) et Cerkovna Ordninga (1564). Le premier est une traduction et une adaptation de la Confession d'Augsbourg et d'autres sources. Le second est le décret ecclésiastique de l'Église évangélique de Carniole, qui représente la première source de droit canon rédigée en langue slovène. En publiant ce décret, Trubar empiète toutefois sur un droit régalien exclusif du prince catholique ("cuius regio, eius religio ») issu de la famille de Habsbourg, qui l'expulse d'Autriche intérieure de façon permanente et définitive en 1565.

Il retourne alors en Allemagne, où il restera jusqu'à sa mort en 1586. Il y est mis en terre par l'un des théologiens évangéliques les plus importants de la deuxième moitié du $16^{\mathrm{e}}$ siècle, Jacob Andreae, l'auteur principal de la Formula concordiae (1577), corédigée entre autres avec Martin Chemnitz. Andreae prononça un éloge funèbre (Leichpredig) devant la tombe de Trubar, et sa famille finança l'érection d'une grande épitaphe en son honneur, que l'on peut encore admirer aujourd'hui, à un endroit bien en vue de l'église de Derendingen. Il s'agit là de la seule représentation artistique de la famille de Trubar (celui-ci a eu cinq enfants de trois épouses, dont trois qui lui ont survécu - ses fils Primož et Felicijan, et sa seule et unique fille). En 1586, Andreae écrivit par ailleurs un livre basé sur son éloge funèbre, dans lequel il « canonise » Trubar, le situant parmi les principaux théologiens européens de la deuxième génération après Luther. L'ouvrage d'Andreae est publié en 1588 (par Matija Trost) aussi dans une traduction slovène, considérée comme la première occurrence du genre biographique dans cette langue.

Les travaux de Trubar constituent le premier écrit d'importance de la littérature slovène et l'œuvre la plus célèbre rédigée en cette langue, devant celle d'Ivan Cankar (écrivain représentant du modernisme de la fin $d u 19^{\text {e }}$ siècle). L'édition scientifique et critique du corpus littéraire Collected Works of Primož Trubar (commencée en 2002, avec 11 ouvrages parus jusqu'à présent, disponibles en ligne à partir du tome 6 à l'adresse http://www.pei.si/Sifranti/StaticPage. aspx?id=86) compte plus de 8000 pages. Outre son volume, il convient de noter également le contenu et la diversité de genres de l'œuvre de Trubar. Il a ainsi écrit et publié : trois abécédaires $(1550,1555,1566)$; trois petits catéchismes $(1550,1555,1567)$; un grand catéchisme destiné au clergé (1575); deux postilles ${ }^{2}$ (1558 et 1595, cette dernière étant la traduction de la Hauspostille de Luther - Trubar a mis un point final à ses travaux, publiés à titre posthume, 14 jours avant sa 
mort) ; six livres de poésie, dont deux recueils complets de chants religieux, qu'il considérait comme sa propre création $(1563,1567,1574,1575,1579)$; une traduction et adaptation de la Confession d'Augsbourg (1562) ; un décret ecclésiastique (1564); plusieurs préfaces, parmi lesquelles la «Longue préface » (1557), le tout premier essai - ou traité - scientifique et théologique en langue slovène, tandis que les autres marquent le début de l'historiographie et de la musicologie dans la culture slovène (en particulier les préfaces d'Articuli, 1562, et des Psaumes complets de David, 1565) ; de nombreuses traductions de segments de la Bible (1555-1579, 1581/1582), parmi lesquelles trois versions de l'Évangile de Matthieu, deux versions du Nouveau Testament et une version des Psaumes (1565). Les écrits de Trubar, principalement rédigés en slovène et en allemand, furent également traduits et publiés en croate. Le tout premier ouvrage slovène fut imprimé en écriture gothique, tandis que les ouvrages de sciences humaines et les traductions en croate publiés par la suite le furent en cyrillique et en glagolitique. Trubar considérait sa deuxième édition révisée du Nouveau Testament (1581/1582) comme son chef-d'œuvre, la réalisation de toute une vie. Conscient de ce fait, il aurait même écrit que «le monde n'a pas vu de tel chef-d'œuvre depuis sa création ». Avec sa traduction du Nouveau Testament et la diligence systématique qu'il apporta à l'éducation des jeunes membres (Dalmatin, F. Trubar, Savinec) de l'« Église de la langue slovène » - l'Église évangélique slovène de Carniole -, Trubar ouvrit la voie à l'essor de la littérature protestante slovène du $16^{\mathrm{e}}$ siècle, qui connut son apogée avec une traduction complète de la Bible, publiée à Wittenberg en 1584, et la première grammaire littéraire slovène, Arcticae Horulae (A. Bohorič) - un «dérivé » de la mise au point linguistique de la Bible dans cette langue. Trubar a jeté ainsi les fondements de la littérature slovène, qui n'a cessé de se développer depuis.

Compte tenu de l'importance des travaux du prêtre évangélique Trubar - rebaptisé par les Allemands le "Luther slovène " - pour l'identité nationale du pays, il apparaît normal que les billets de banque (10 tolars slovènes) de l'État indépendant de la République de Slovénie aient représenté, avant l'introduction de l'euro, les Abécédaires et la seconde édition du Nouveau Testament de Trubar. Les Slovènes ont en effet pu intégrer, grâce à ces deux ouvrages, l'école européenne de la culture : ils ont appris à lire grâce aux Abécédaires, et avec le Nouveau Testament évangélique, ont reconnu leur littérature comme un héritage des grands événements religieux s'étant déroulés sur le territoire allemand.

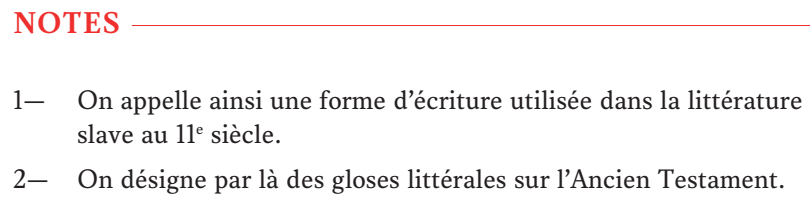

